\title{
Recent Developments in Cryogenic Pulsating Heat Pipes
}

\section{J. M. Pfotenhauer}

Pulsating heat pipes provide an extremely effective method for transferring heat. Several unique features of the cryogenic version of the PHP that have appeared in the recent literature are discussed. These include stable operation with the evaporator in the supercritical region, a temperature dependent fill ratio, and a length invariant conductance for the helium PHP. Results for helium, neon, hydrogen, and nitrogen PHPs are included along with performance dependence on orientation, fill level, and number of parallel tubes.

Keywords: Pulsating heat pipe; Cryogenic PHP; Heat transfer

Received 29 December 2018

DOI: $10.30919 /$ esee $8 \mathrm{c} 215$

An increasing number of research groups and associated publications are emerging in the field of cryogenic pulsating heat pipes. This passive, yet highly effective, heat transfer device has been extensively explored in the room temperature range since the mid 1990s, however its performance with cryogenic fluids has been investigated for less than ten years. In its most common configuration, a capillary tube extends back and forth multiple times between a heat source and a heat sink, forming a closed loop passage. The volume defined by the inside of the capillary tube is partially filled with a fluid, while the remaining volume is occupied by the saturated vapor. The inside diameter of the capillary is sufficiently small so that surface tension forces exceed gravity forces, and the liquid and vapor regions are randomly distributed throughout the length of the capillary tubing. Heat applied at the evaporator region and removed at the condenser region gives rise to spontaneous pressure driven motion of the liquid and vapor regions, motion that effectively transfers heat from the evaporator to condenser regions.

In the cryogenic regime, features of the PHP are attractive for applications related to cryocooled superconducting magnets, space applications, and ground transportation of cryogens. Cryocooled superconducting magnets may benefit from the large heat transfer properties of a PHP to accelerate cooldown from room temperature, to enhance the effectiveness of re-condenser systems, and to efficiently distribute heat over large regions of the magnets without massive conduction bus-bars. In space applications, both the effective heat transfer properties and the low mass of the PHP represent attractive features. Furthermore, the problem of thermal roll-over in ground transported cryogen tanks could be addressed for the industrial gas industry.

Multiple early reports from a Japanese collaboration ${ }^{1,2}$ identified the potential of PHPs for cooling superconducting magnets and explored the behavior of pulsating heat pipes using hydrogen, neon, and nitrogen. Defining an effective thermal conductivity according to Fourier's law and based on $\mathrm{q}$, the total heat transferred between the evaporator and condenser regions, $T_{e}-T_{c}$, the temperature difference between the evaporator and condenser ends, $\mathrm{L}$, the length of the adiabatic region between the evaporator and condenser regions, and $\mathrm{A}$, the total cross

Department of Mechanical Engineering, University of WisconsinMadison, USA

*E-mail: pfot@engr.wisc.edu sectional area of the fluid involved in the heat transfer, the Japanese group reported surprisingly large effective thermal conductivity values for the fluids. Values up to $18 \mathrm{~kW} / \mathrm{m} \cdot \mathrm{K}, 20 \mathrm{~kW} /(\mathrm{m} \cdot \mathrm{K})$, and 12 $\mathrm{kW} /(\mathrm{m} \cdot \mathrm{K})$ were reported for nitrogen, neon, and hydrogen respectively. The same reports also presented a significant dependence of the effective conductivity on the orientation of the tubes with respect to gravity. Although Fourier's law is an inappropriate equation to describe the heat transfer resulting from such a dynamic two-phase flow phenomenon, it is nevertheless frequently used as a benchmark to describe the thermal performance of the PHP.

In general, the performance of a PHP depends on three groups of factors: fluid properties, configuration, and operation parameters. Significant fluid properties include specific heat, latent heat, viscosity as well as many others. Examples of configuration factors include tube diameter, lengths of the evaporator condenser and adiabatic sections, and number of turns. Operational parameters include factors such as fill ratio, orientation, and heat load as well as others.

During the past year a number of significant PHP performance features unique to the cryogenic fluids have appeared in the literature. In the first of these, ${ }^{3}$ the fill ratio is shown to be a temperature dependent property, even for a sealed PHP volume. Because the critical pressure and critical temperature for many of the cryogenic fluids can be easily exceeded, operating the PHP over a reasonable range of applied heat moves the thermodynamic state of the PHP throughout the vapor dome. For a fixed mass and fixed volume, and therefore a fixed specific volume, increasing the saturation temperature significantly modifies the fill ratio. If the specific volume of the PHP is less than the critical specific volume, an increase in temperature (and pressure) increases the liquid fraction. On the other hand, if the specific volume is larger than the critical specific volume, an increase in temperature (and pressure) results in an increase of vapor, leading to dry-out conditions for the PHP. The initial fill ratio therefore enables the operator to determine both the limiting PHP temperature and its behavior at the high heat load conditions.

A second surprise, closely related to the first, has been reported by two independent research groups. ${ }^{5,6}$ Whereas for all other fluids, heat loads that drive the evaporator beyond saturation conditions result in dry-out and thermal run-away, for helium the PHP continues to operate with oscillatory behavior even when the evaporator temperature is well above the critical temperature. (Fig. 1) In one study ${ }^{5}$ the evaporator reached $20 \mathrm{~K}$ while the PHP continued its usual behavior, that is without a thermal run-away. 


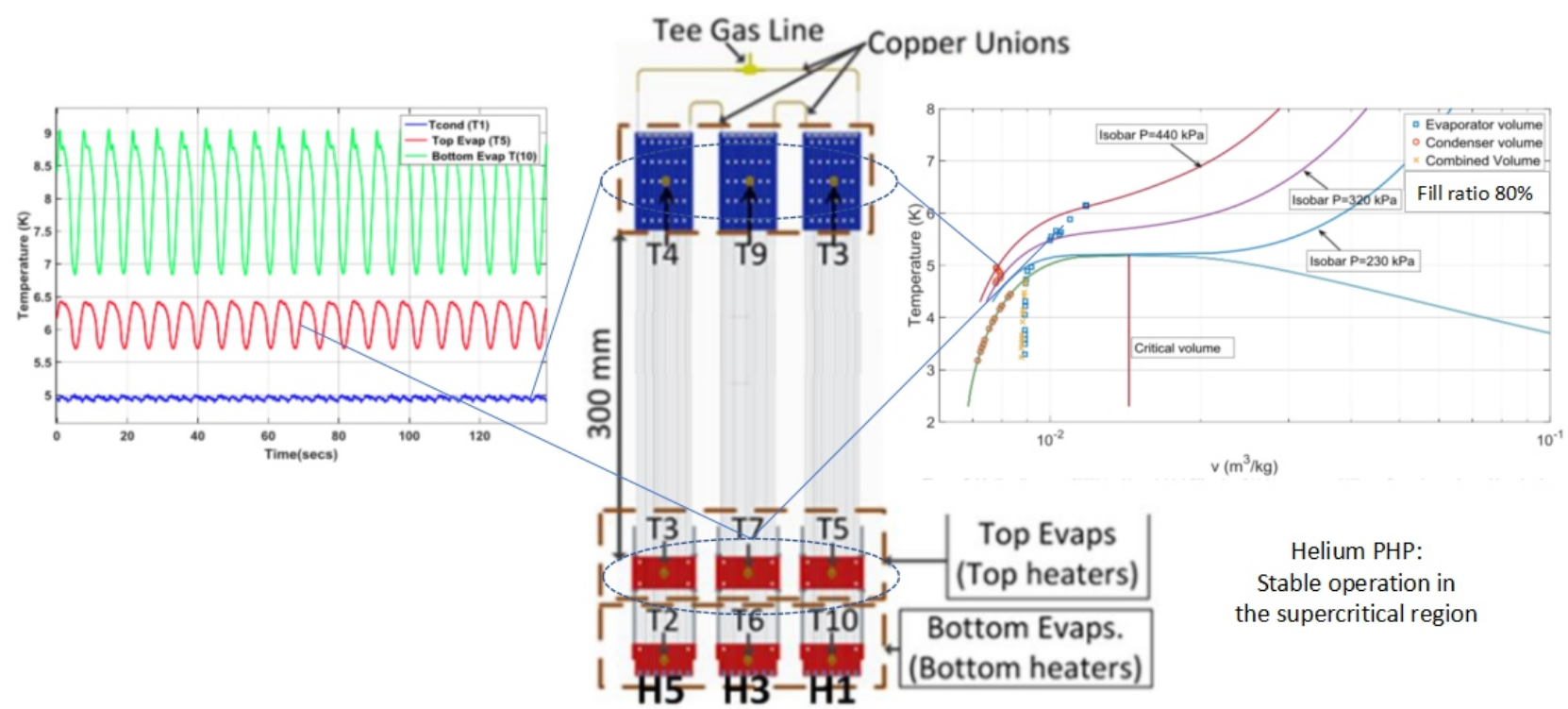

Fig. 1 Helium PHP with the evaporator temperature exceeding the critical temperature. Left: temperature oscillations in the bottom evaporator (green), top evaporator (red) and condenser (blue) regions. Middle: Geometric configuration of 3 identical and serially connected 14-tube PHPs. Condenser at top (blue), $300 \mathrm{~mm}$ long adiabatic section, top and bottom evaporator heaters (red), and thermometer locations (T2-T10). Right: T-v plot showing thermodynamic location of condenser and evaporator regions.

The third surprising behavior is also associated with a helium PHP. $^{4}$ In this case, when the adiabatic length of the PHP was increased from $0.3 \mathrm{~m}$ to $1 \mathrm{~m}$, an identical heat load produced an identical temperature difference between the evaporator and condenser. For heat transfer systems obeying Fourier's law such a change would increase the temperature difference by a factor of 3.33. The imperceptible temperature change demonstrated by the helium PHP resulted in an increase of the 'effective' thermal conductivity from $50 \mathrm{~kW} /(\mathrm{m} \cdot \mathrm{K})$ to $142 \mathrm{~kW} /(\mathrm{m} \cdot \mathrm{K})$. Clearly, additional measurements are desired to determine the extent of adiabatic lengths over which such behavior can be maintained.

The optimum fill ratio for the various cryogenic fluids has been reported by multiple groups. In each case, the 'optimum' value is defined as that which produces the maximum effective thermal conductivity. In a study using normal hydrogen contained in a $2.3 \mathrm{~mm}$

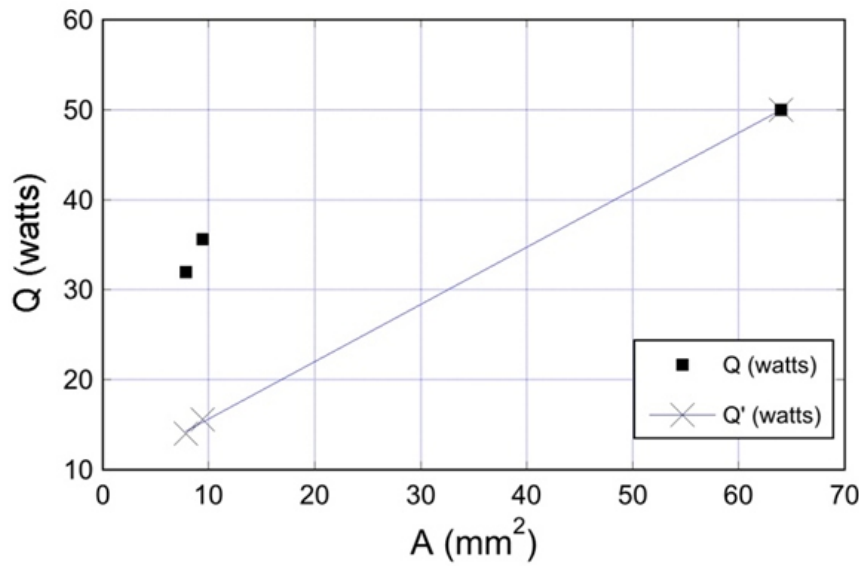

Fig. 2 Applied heat limit for Neon PHP vs. the combined crosssectional area of the parallel tubes. Q values represent direct measurements, Q' values are all adjusted for the values in a horizontal configuration.
ID capillary with respective evaporator, adiabatic, and condenser regions of $50 \mathrm{~mm}, 500 \mathrm{~mm}$, and $50 \mathrm{~mm}$ serially connected over four parallel lengths of tubing, ${ }^{6}$ the optimum fill ratio is observed to depend on the heat load. For heat loads less than 1 watt, the optimum fill ratio is $10 \%$. For heat loads between 1 and 2.8 watts, the optimum fill ratio is between 20 and $28 \%$, while for heat loads larger than 2.8 watts, the optimum fill ratio is in the $40 \%$ range. The data suggest different types of heat transfer mechanisms are active in the various heat load ranges. Investigations with neon ${ }^{7-9}$ and nitrogen ${ }^{10}$ reveal optimum fill ratios of $\sim 30 \%$ and $\sim 45 \%$ respectively. With helium an optimum fill ratio is found between $60 \%$ and $70 \%$. Interestingly, one investigation ${ }^{5}$ reveals that the optimal effective conductivity is larger for an 8-tube configuration as compared to a 48-tube helium PHP.

Similar to many of the room temperature studies, those using cryogenic fluids uniformly report that the thermal conductance increases with the applied heat load up to the optimal value, beyond which it falls precipitously. For each of the studies using hydrogen or neon, the precipitous drop is associated with the evaporator temperature exceeding the fluid's critical temperature and a corresponding thermal run-away. Investigations into the influence of orientation with respect to gravity $^{1,2}$ also mirror those with room temperature fluids showing significant degradation as the axis between the evaporator and condenser changes from vertical (heating from below) toward horizontal. However, unlike the room temperature studies, degradations by roughly a factor of two occur going from vertical to horizontal even when the PHP is comprised of more than 40 parallel tubes.

Also, in common with room temperature fluids, the specific limit of applied heat load for neon is shown to increase with the number of parallel tubes. From multiple studies ${ }^{7-9}$ one finds the limiting heat load increasing from 32 watts with a combined tube area of $\mathrm{A}=7.85 \mathrm{~mm}^{2}$ to 35.6 watts with $\mathrm{A}=9.42 \mathrm{~mm}^{2}$, to 50 watts with $\mathrm{A}=64 \mathrm{~mm}^{2}$. It is significant to note that the PHP orientation for first two of these reports was vertical while for the last it was horizontal. Fig. 2 displays the applied heat limit as a function of the combined cross-sectional area of all the tubes in the PHP. When the heat load limit for the two smallest areas is adjusted to the expected value of the horizontal orientation as 
suggested by ${ }^{1}$ the three data points define a linear relationship between the applied heat load limit and the total cross-sectional area of the PHP.

For hydrogen ${ }^{6,11}$ the limiting heat load remains between 9 and 10 watts with $\mathrm{A}=16.6 \mathrm{~mm}^{2}$ and $\mathrm{A}=41.5 \mathrm{~mm}^{2}$. In both cases, the condenser temperature reaches $27 \mathrm{~K}$ when the evaporator temperature reaches $33 \mathrm{~K}$. Studies using helium with varying number of tubes report similar limiting applied heat loads of approximately 1 watt. However, the evaporator and condenser temperatures respectively reach $24 \mathrm{~K}$ and $4.8 \mathrm{~K}$ with the 8-tube configuration while they only reach respective values of $5.2 \mathrm{~K}$ and $4.6 \mathrm{~K}$ with the 42-tube configuration, and $5.5 \mathrm{~K}$ and $4.75 \mathrm{~K}$ with the 48-tube configuration. A full characterization of the heat transfer limits for each of the cryogenic fluids as a function of orientation and the number of tubes has yet to be completed.

\section{Summary}

Cryogenic pulsating heat pipes present a number of unique features such as a temperature dependent fill ratio. Helium PHPs provide steady operation in the supercritical regime, and over at least a limited range of adiabatic lengths demonstrate a length invariant conductance. Furthermore, data with helium PHPs suggest that the optimal effective conductivity depends on the number of turns, with an 8-tube configuration preferred over a 48-tube configuration. Many performance features have yet to be fully explored, but those revealed to date provide exciting possibilities for the superconducting magnet and aerospace applications.

\section{References}

1. T. Mito, K. Natsume, N. Yanagi, H. Tamura, T. Tamada, K. Shikimachi, N. Hirano and S. Nagaya, IEEE Trans. Appl. Supercond., 2011, 21(3), 2470-2473.

2. K. Natsume, T. Mito, N. Yanagi, H. Tamura, T. Tamada, K. Shikimachi, N. Hirano and S. Nagaya, Cryogenics, 2011, 51, 309-314.

3. X. Sun, J. Pfotenhauer, B. Jiao, L. D. Fonseca, D. Han and Z. Gan, Int. J. Heat Mass Tran., 2018, 126, 237-244.

4. L. D. Fonseca, J. M. Pfotenhauer and F. K. Miller, Int. J. Heat Mass Tran., 2018, 123, 655-656.

5. M. Li, L. Li and D. Xu, Cryogenics, 2018, 96, 159-165.

6. X. Sun, S. Li, B. Jiao, Z. Gan and J. Pfotenhauer, Cryogenics, 2019, 97, 63-69.

7. Q. Liang, Y. Li, Q. Wang, Cryogenics, 2018, 89, 102-106.

8. Q. Liang, Y. Li, Q. Wang, Heat Mass Transfer, 2018, 54 (6), 1721-1727.

9. M. Barba, R. Bruce, F. Bouchet, A. Bonelli, B. Baudouy, paper E-14:073 presented at the 2018 International Cryogenic Engineering Conference, Oxford UK, September (2018).

10. R. Bruce, M. Barba, A. Bonelli and B. Baudouy, Cryogenics, 2018, 93, 66-74

11. H. R. Deng, Y. M. Liu, R. F. Ma, D. Y. Han, Z. H. Gan and J. M. Pfotenhauer, IOP Conf. Series: Materials Science and Engineering, 2015, 101, 012065. 